\title{
ZUR VERBREITUNG UND GEOGRAPHISCHEN BEDEUTUNG DER GRÜNHECKEN IN DER SCHWEIZ*
}

\section{Ingrid Steiner-Haremaker und Dieter Steiner}

Die Grünhecke ist ein Objekt in der Agrarlandschaft, das für die geographische Forschung nicht ohne Bedeutung ist. In vielen Landstrichen Europas, so vor allem im atlantischen Bereich, kommen Hecken in einer derartigen Dichte vor, daß sie nicht nur zu einem auffallenden, sondern geradezu zum physiognomisch dominanten Bestandteil des Landschaftsbildes werden.

Diese Regionen sind denn auch in der Literatur verschiedentlich beschrieben worden (Hartke (16), Jessen (20), Juillard und Meynier (22), Kuhn (24), Lautensach (25), Lebon (27), Marquardt (29), Timmermann (40), Troll (41), Uhlig (43)).

In der Schweiz treten heute die Grünhecken selten und nur auf kürzere Strecken in so großer Zahl auf, da $B$ man von einer «Heckenlandschaft» sprechen könnte. Es können aber auch vereinzelt vorkommende Hecken oder Heckennetze infolge ihrer Entstehung oder Funktion von geographischer Bedeutung sein. Es soll deshalb im folgenden näher auf das Vorkommen und den Aussagewert der Hecken im Raume der Schweiz eingegangen werden, von denen im gröBeren Zusammenhang so gut wie nichts bekannt ist. Wir untersuchen dabei zunächst die Verbreitung und die Häufigkeit der Hecken, um zu einer Vorstellung zu kommen, wo und in welchem Maße wir sie überhaupt zu erwarten haben.

\section{Die Heckendichte und die Regionen stärkster Konzentration}

Die kartographische Darstellung der Grünheckendichte (Heckenlänge pro Fläche), wie sie in Fig. 1 präsentiert wird, ergibt eine Übersicht über die Gebiete, in denen heute Hecken häufig anzutreffen sind. Bei der Besprechung dieser Darstellung nehmen wir vorläufig keine Rücksicht auf die Art der Hecken. Auf diesen Punkt treten wir im zweiten Abschnitt ein.

Die Grundlage für Fig. 1 bildete die Landeskarte der Schweiz, auf der die Hekken mit einer speziellen Signatur wiedergegeben sind. Wir verwendeten die Blätter $1: 25000$ für Jura, Mittelland, Alpenrand und Südtessin und die Blätter 1 : 50000 für das Alpengebiet ${ }^{1}$. Daraus wurde zunächst eine Gitternetzkarte erstellt, indem fortlaufend in Quadraten von $2 \mathrm{~km}$ Seitenlänge die Länge der dargestellten Hecken auf $100 \mathrm{~m}$ genau geschätzt und durch die Fläche der potentiellen Heckenstandorte (Gesamtfläche minus Siedlungen, Wald, Gewässer und Hochgebirge) dividiert wurde. Die aus der Karte 1:50000 gewonnenen Werte multiplizierten wir gemäß dem stärkeren Generalisierungsgrad mit einem Korrekturkoeffizient. Die hier gezeigte Dichtekarte stellt eine starke Generalisierung der erwähnten Gitternetzkarte dar: Sie faßt unter Vernachläßigung einzelner Quadrate gleiche Dichten zu größeren zusammenhängenden Flächen zusammen. Außerdem haben wir uns auf die Wiedergabe von 3 Dichtestufen beschränkt, nämlich 1) 0-500 m Heckenlänge pro km² ;2) $510-2000 \mathrm{~m}$; 3) $2010 \mathrm{~m}$ und mehr ${ }^{2}$. Wir erhalten damit eine Karte, die nur die Gebiete stärkster Konzentration hervortreten läßt. Auf Detailprobleme der angewendeten Methode und auf die Frage des Generalisierungsgrades auf der Karte 1:25000 können wir hier nicht weiter eintreten. Dafür sei auf die Originalarbeit verwiesen (38). Wir dürfen

* Der vorliegende Beitrag stützt sich zur Hauptsache auf eine von der Autorin am Geographischen Institut der Universität Zürich 1959 abgeschlossene Diplomarbeit (38). Ergänzende Angaben sammelten wir nachträglich aus weiteren Feldbegehungen und aus dem Studium von Luftbildern.

1 Das mit der Karte 1:25 000 bearbeitete Gebiet läßt sich aus dem Publikationsstand vom Oktober 1958 ersehen. Alle damals bestehenden Blätter mit Ausnahme von 1197 (Davos), 1257 (St. Moritz) und 1313 (Bellinzona) fanden Verwendung.

2 Auf der Originalkarte (38) sind 6 Stufen dargestellt. 


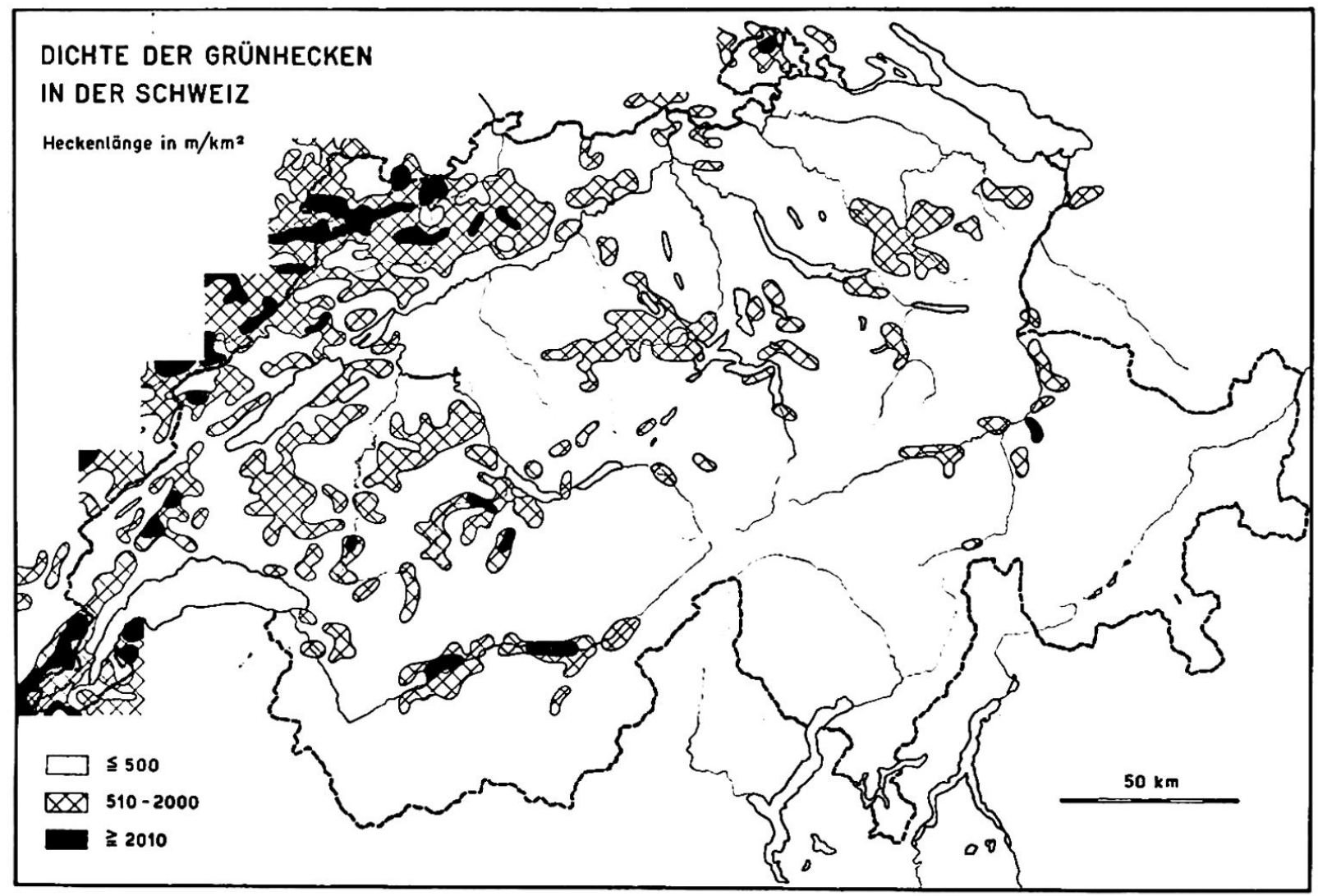

Fig. 1. Grünheckendichte in der Schweiz auf Grund der Landeskarte 1:25000 (Jura, Mittelland, Alpenrand und Südtessin) und 1:50000 (Alpen). Um eine einheitliche Darstellung zu erreichen, sind die aus der Karte 1:50000 gewonnenen Werte mit Hilfe eines mittleren Korrekturfaktors auf die wahrscheinlichen Werte im Maßstab 1:25000 umgerechnet (vgl. Text).

aber auf alle Fälle annehmen, daß wir mit unserem Verfahren wenn auch nicht absolut zuverläßige Angaben so doch relativ gut vergleichbare Resultate erhalten haben.

Auf Fig. 1 machen sich nun folgende Gebiete mit großen Heckendichten bemerkbar: 1) Einzelne Teile des Tafeljuras und der Randen;2) Die westliche Hälfte der Ajoie; 3) Der größte Teil des Kettenjuras mit einer großen zusammenhängenden Fläche hoher Dichte östlich der Linie Biel-Freiberge; 4) Einzelne Regionen des westlichen Mittellandes, so die Umgebung von Genf, die Jurafuß-Zone von der westlichen Landesgrenze bis gegen Yverdon und das waadtländische und freiburgische Hügelland südlich des Neuenburgersees; 5) Eine subalpine Zone, die sich in voneinander getrennte Gebiete stärkerer Konzentration gliedert: Das Gebiet rund um Bulle und südlich von Freiburg, das Schwarzenburgerland südwestlich von Bern, das Emmental, das Entlebuch und das Gebiet zwischen Luzern und dem Sempachersee, das Zürcher Oberland östlich des Tößtales, die Ricken-Region und das mittlere Toggenburg und das Gebiet um St. Gallen und Trogen; 6) Einige randalpine Täler, nämlich das Tal der Sarne südlich von Bulle, das Simmental, die Umgebung von Schwyz mit dem Muotatal, eine Region südwestlich von Lachen, das Glarner Linthtal und das Churer Rheintal; 7) Einzelne inneralpine Täler wie der mittlere Abschnitt des Walliser Rhonetals, das Visper Tal, das Vorderrheintal und das Domleschg.

Lokal finden wir die größten Heckendichten bei Les Bayards (NE) mit $5,5 \mathrm{~km}$ Länge pro $\mathrm{km}^{2}$, im Randengebiet mit $4,6 \mathrm{~km}$, am waadtländischen Jurafuß bei Bière mit $3,8 \mathrm{~km}$, in der Umgebung von St. Ursanne mit 3,6 km, rund um Moutier mit 3,3 km und in der westlichen Ajoie mit $3,1 \mathrm{~km}$. Vergleichsweise betragen die Dichten im von MarQuARdT (29) untersuchten Knickgebiet Schleswig-Holsteins 1 bis über $10 \mathrm{~km}$ Länge pro $\mathrm{km}^{2}$. Interessant ist auch das häufigere Vorkommen hoher Dichten beim Überschreiten der westlichen Landesgrenze auf französischem Boden. 


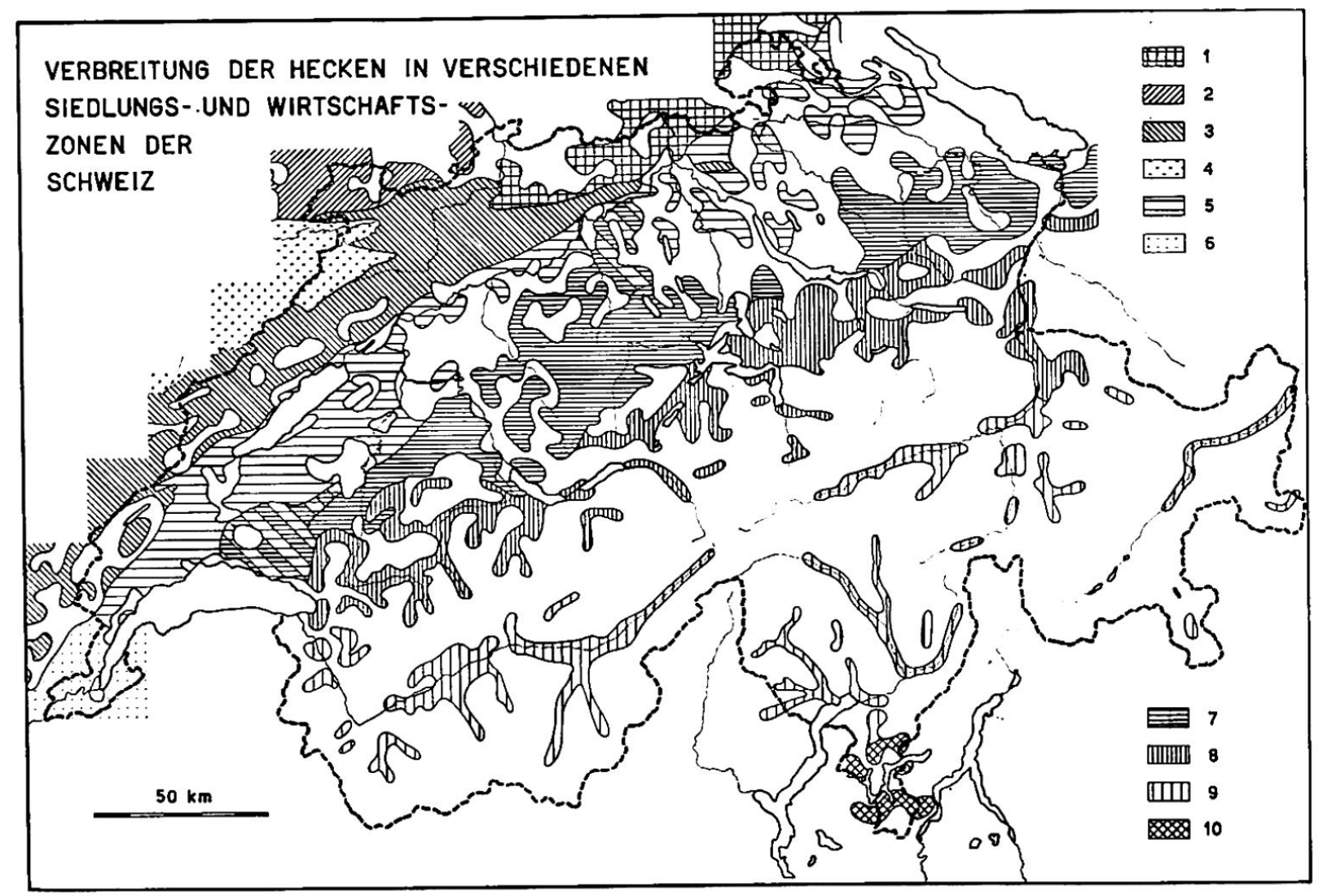

Fig. 2. Verbreitung der Grünhecken in verschiedenen Siedlungs- und Wirtschaftszonen der Schweiz. Es bedeuten: $\mathbf{1}=$ Tafeljura und Randen: Vorwiegend Dorfsiedlung, Wildhecken auf Rainen und Lesesteinriegeln in heutigem und ehemaligem Acker- und Rebland; $2=$ Ajoie: Dorfsiedlung, Wildhecken auf Lesesteinen und Ackerrainen, Bocage-Komplexe in Grünland, Schutzhecken entlang größerer Weideflächen; $3=$ Kettenjura: In den Tälern Dorfsiedlung, hie und da Reste von Grünland-Bocage, Hecken an der Grenze von Allmendweiden, als Besonderheit Wildhecken von Les Bayards; auf den Höhen Streusiedlung mit meist gut arrondierter Blockflur, Hecken zur Abgrenzung der Wirtschafts- und Nutzungsareale, im östlichen Teil vorwiegend Kulturhecken, im westlichen wildes Gebüsch auf Trockenmauern; $4=$ Plateaujura (Freiberge): Nebeneinander von geschlossener und Streusiedlung, verschiedener Grad der Arrondierung, Wildhecken auf Trockenmauern als Markierung von Besitz- und Nutzungsgrenzen, gelegentlich Lesesteinriegel mit Gebüsch in heutigem Wiesland; $5=$ Mittelland: Dorfsiedlung mit einstiger Dreizelgenwirtschaft, geringe Reste einer früheren Heckenlandschaft, hauptsächlich Wildhecken auf Ackerrainen; $6=\mathrm{Kt}$. Genf: Dorfsiedlung mit früherer Zweifelderwirtschaft, Reste einer Halbbocage; 7 = Subalpine Zone: Streusiedlung mit früherer Koppelwirtschaft, Kulturhecken auf den Besitzgrenzen als Reste einer Vollbocage; $8=$ Randalpine Täler: Vorwiegend geschlossene Siedlung, Hecken hauptsächlich um Wiesenparzellen mit Außenställen, zum Teil wild auf Mäuerchen und Lesesteinen (auf Schuttkegeln); $9=$ Inneralpine Täler: Dorfsiedlung, Ackerland an terrassierten Hängen, Wildhecken zwischen den Parzellen, auf den Böschungen oder Mäuerchen, seltener in Grünland; $10=$ Südtessin : Dörfer, gelegentlich Hecken auf Mäuerchen, zum Teil zur Abgrenzung von größeren Weidearealen.

\section{Die Verbreitung der verschiedenen Heckentypen im Zusammenhang mit natur- und kulturgeographischen Voraussetzungen}

Wir weiten die Betrachtung in diesem Abschnitt von den Zonen stärkster Heckendichte auf die gesamten Verbreitungsgebiete aus und untersuchen die verschiedenen Heckentypen. Wir beschränken uns dabei auf die in oder um Acker- und Grünland auf Parzellengrenzen wachsenden Hecken und unterscheiden zunächst Wild- und Kulturhecken. Wildhecken sind spontan auf Standorten entstanden, die von der menschlichen Nutzungstätigkeit nicht erreicht werden, wie Ackerraine, Lesestein riegel und Trockenmauern. Kulturhecken sind absichtlich gepflanzt worden und üben somit eine bestimmte Funktion aus oder haben eine solche zum mindesten in früherer Zeit ausgeübt. Die zweite Gruppe kann weiter in Hecken auf Besitzgrenzen und Hecken 
auf Nutzungsgrenzen (auf der einen Seite stets Weide in irgendeiner Form) eingeteilt werden. Die beiden Funktionen können aber auch zusammenfallen ${ }^{3}$.

Die als Fig. 2 gezeigte Karte gibt nun die effektive Verbreitung der Hecken wieder, wobei eine Aufteilung in verschiedene Zonen vorgenommen wurde. Diese lehnt sich an eine großräumige morphologisch-klimatische, damit aber gleichzeitig auch anthropogeographische Gliederung der Schweiz an. Jede dieser Zonen weist in sich cine gewisse Gleichartigkeit inbezug auf Siedlungs-, Wirtschafts- und Flurformen auf. Da der Typ der Hecken mit der wirtschaftlichen Struktur eng verknüpft ist, ist eine derartige Einteilung gegeben.

Im Bereich des mit Karte 1:25000 bearbeiteten Gebietes wählten wir als Verbreitungsgrenze die Linie, an der die Dichte auf $100 \mathrm{~m} / \mathrm{km}^{2}$ sinkt. Bei kleineren Werten verschwinden die Hecken praktisch aus dem Landschaftsbild. In der alpinen Region ermittelten wir die ungefähre Verbreitung auf Grund von Feldbeobachtungen, Karten, Literatur und Luftbildern ${ }^{4}$.

Im folgenden besprechen wir die ausgeschiedenen Zonen einzeln, wobei wir uns an die in Fig. 2 gegebene Numerierung halten:

1) Tafeljura und Randen: In diesem Gebiet sind die Lesesteinriegel stark verbreitet. Sie sind im Laufe der Zeit an den Parzellengrenzen durch die Ablagerung von Sammelsteinen entstanden, die beim Pflügen der flachgründigen und skelettreichen Kalkböden immer wieder anfielen. Sie finden sich vor allem an den steilen Talhängen - zum Teil quer, zum Teil parallel zum Gefälle - in heutigem oder einstigem Ackerund Rebland der Dorfwirtschaftsgebiete. Allmählich sind sie von wildem Gebüsch überwuchert worden, sodaß sich jetzt zwischen den Parzellen oft Hecken erstrecken (vgl. Abb. 1). Die Steinhaufen liegen heute häufig in Wiesland oder sogar im Wald und lassen eine in früherer Zeit bedeutend größere Ausdehnung des Acker- und Reblandes erkennen (vgl. WYDER für den Randen (52) und Vosseler für den Aargauer Tafeljura (46) 5. Wildhecken stocken häufig auch auf Ackerrainen (Terrassenböschungen zwischen zwei hangparallelen Parzellen), wobei aber auch hier das Terrain heute oft von Grünland eingenommen ist. Wo die Hecken auch jetzt noch Ackerparzellen begleiten, kann man beobachten, da $\beta$ die Bauern das Gebüsch als unerwünschten Schattenspender gelegentlich roden oder abbrennen. Bei Güterzusammenlegungen werden Sammelsteine und Hecken mitunter ganz beseitigt.

2) Ajoie: Die Ajoie zeigt inbezug auf die Genese der agrarischen Struktur und die damit zusammenhängenden Hecken einige Besonderheiten, die eine gesonderte Besprechung dieses Gebietes rechtfertigen. Die Hecken nehmen in dieser Region mit vorwiegender Dorfsiedlung und ehemaliger Dreizelgenwirtschaft im wesentlichen drei verschiedene Standorte ein: a) Lesesteinhaufen und Ackerraine, analog zum Tafeljura (z.B. in der Gegend von Bure, vgl. NerTz [32]); b) Parzellengrenzen in unregelmäßig blockartig aufgeteilten Komplexen von Grünland (Wiese oder Weide, z. B. in der Umgebung von Rocourt und Grandfontaine, vgl. Abb. 2) ; c) Die Nutzungsgrenze zwischen dem Kulturland i. e.S. und größeren Allmendweiden, wobei diese mit den

3 Auf andere seltenere Heckentypen, die nicht auf Parzellengrenzen wachsen oder aber eine andersartige Funktion ausüben, wie heckenartige Gebüschstreifen auf Anstehendem, Erosionsschutz-Hecken, Windschutzstreifen und Grünstreifen entlang von Straßen sei hier nur am Rande hingewiesen.

4 Als Ergänzung bei der Herstellung der Verbreitungskarte konnten wir die Karten von Escher (10) (volkskundlich, ganze Schweiz), und von Hager (15) und SPINner (37) (pflanzengeographisch, Vorderrheintal, resp. Neuenburger Jura) heranziehen.

5 Zum Teil finden sich die Lesesteinriegel auch auf der Hochfläche. Die auf der Karte angegebene hohe Dichte auf der Randenhochfläche ergibt sich aber zur Hauptsache aus schmalen Föhrenwaldstreifen, die auf der Landeskarte mit der Heckensignatur dargestellt sind. Diese unechten Hecken (vielfach ohne Unterwuchs) sind durch die Aussaat von Föhrensamen auf schmalen aufgelassenen Ackerparzellen entstanden (vgl. WYDER [52]). 


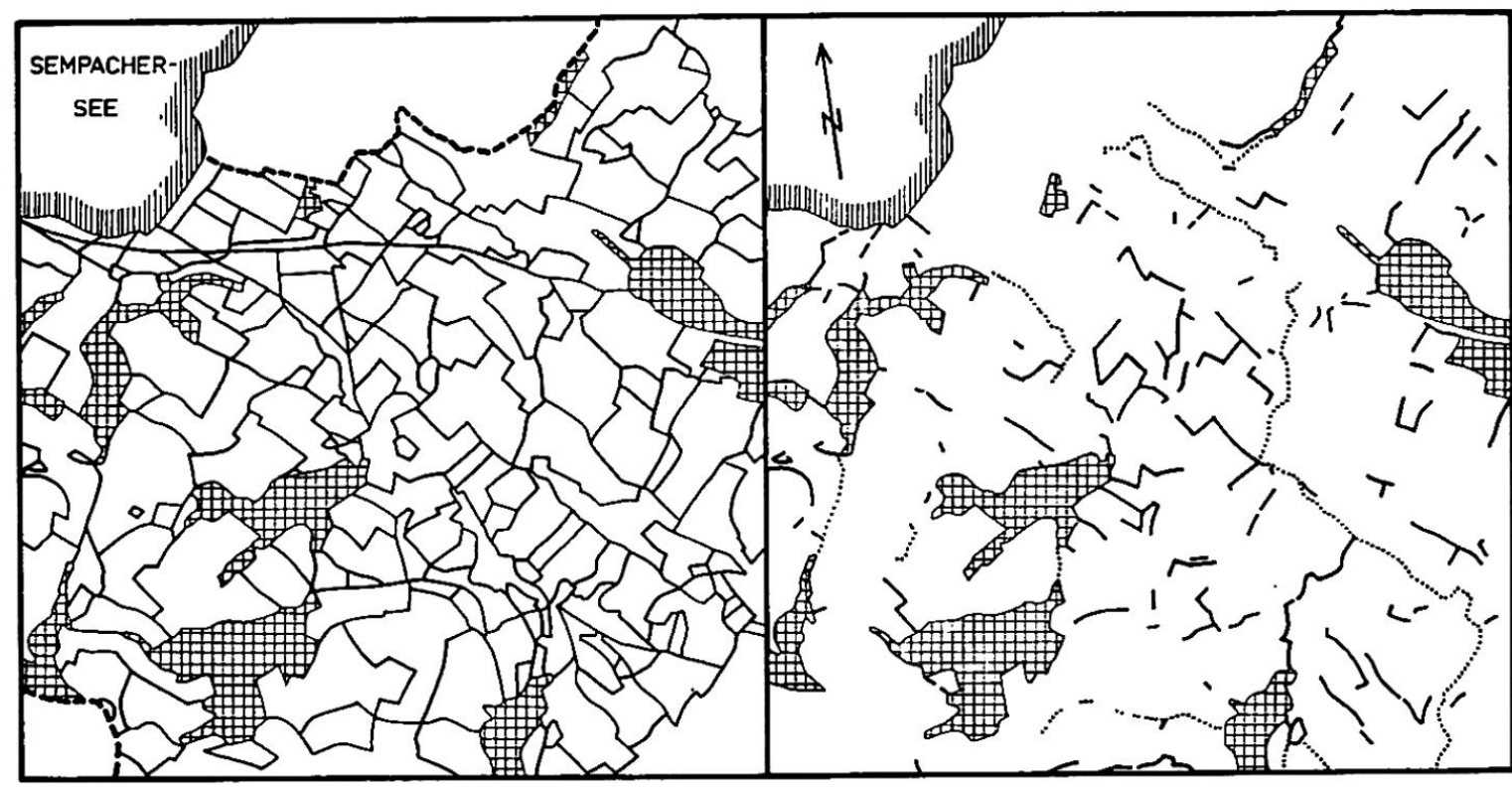

BESITZGRENZEN

GRÜNHECKEN

BACHUFERGEBÜSCH

- GEMEINOEGRENZE WALD

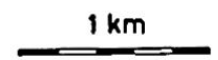

Fig. 3. Auschnitt aus dem Gemeindeareal von Neuenkirch (LU). Links die Einteilung in Besitzparzellen nach dem Grundbuchplan (kleinste Parzellen nicht dargestellt), rechts die Grünhecken auf Grund von Luftbildern der Eidg. Landestopographie. Beispiel für die in Auflösung begriffene Heckenlandschaft der subalpinen Zone.

zugehörigen Dörfern oft mit schmalen, sich trichterförmig öffnenden Viehtriften verbunden sind (z. B. in der Gegend von Courgenay).

Die in Dorfnähe liegenden eingehegten Grünlandkomplexe machen den Eindruck einer Bocagelandschaft im Kleinen. Sie sind vermutlich als Relikte aus der Zeit der Dreizelgenwirtschaft zu erklären. Damals wechselten offene und Bocagekomplexe miteinander ab, worauf wir im dritten Abschnitt näher eintreten werden. Während aber diese Hecken fast überall sonst verschwunden sind, haben sie sich hier teilweise halten können. Dies liegt wohl zum Teil in einem gewissen Konservatismus begründet, läßt sich aber anderseits auch aus der heutigen Nutzung erklären: Während die Weiden früher eher die abgelegenen und schlechteren Standorte einnahmen, hat in neuerer Zeit eine starke Zunahme der Viehwirtschaft dazu geführt, daß häufig gutes, in Dorfnähe liegendes Kulturland als Weide genutzt wird (vgl. NerTz [32]). Damit üben die Hecken noch eine sichtbare Funktion aus. Das läßt sich auch daraus ersehen, daß, im Gegensatz zu andern Gebieten, die Hecken noch eine gewisse Pflege erfahren, indem etwa Lücken durch knickartige Verflechtungen ausgebessert werden. Das beschriebene Landschaftsbild setzt sich über die Landesgrenze ins südliche Elsaß fort.

Auf der andern Seite sind die ehemaligen Weideflächen heute zum Teil wieder aufgeforstet oder sie werden anderweitig agrarisch genutzt. So lassen sich etwa Fälle beobachten (z. B. bei Courgenay), in denen Acker- und Wiesenparzellen in dem vom Dorf ausgehenden Viehtrieb liegen. Die Hecken stehen aber beidseits noch und spiegeln den alten $Z$ ustand wieder.

3) Kettenjura: Wir haben innerhalb des Kettenjuras mit zwei grundlegend verschiedenen Siedlungs- und Wirtschaftszonen zu rechnen, die sich aber in gesetzmäßiger Weise ablösen: In den Tälern finden wir Dorfsiedlungen und stärkeren Anteil des Ackerbaus, auf den Höhen Einzelhofsiedlungen mit dominanter Viehwirtschaft (Jurabetriebe mit und ohne Weide, Sömmerungsbetriebe). Die Dorfgebiete zeigen inbezug 

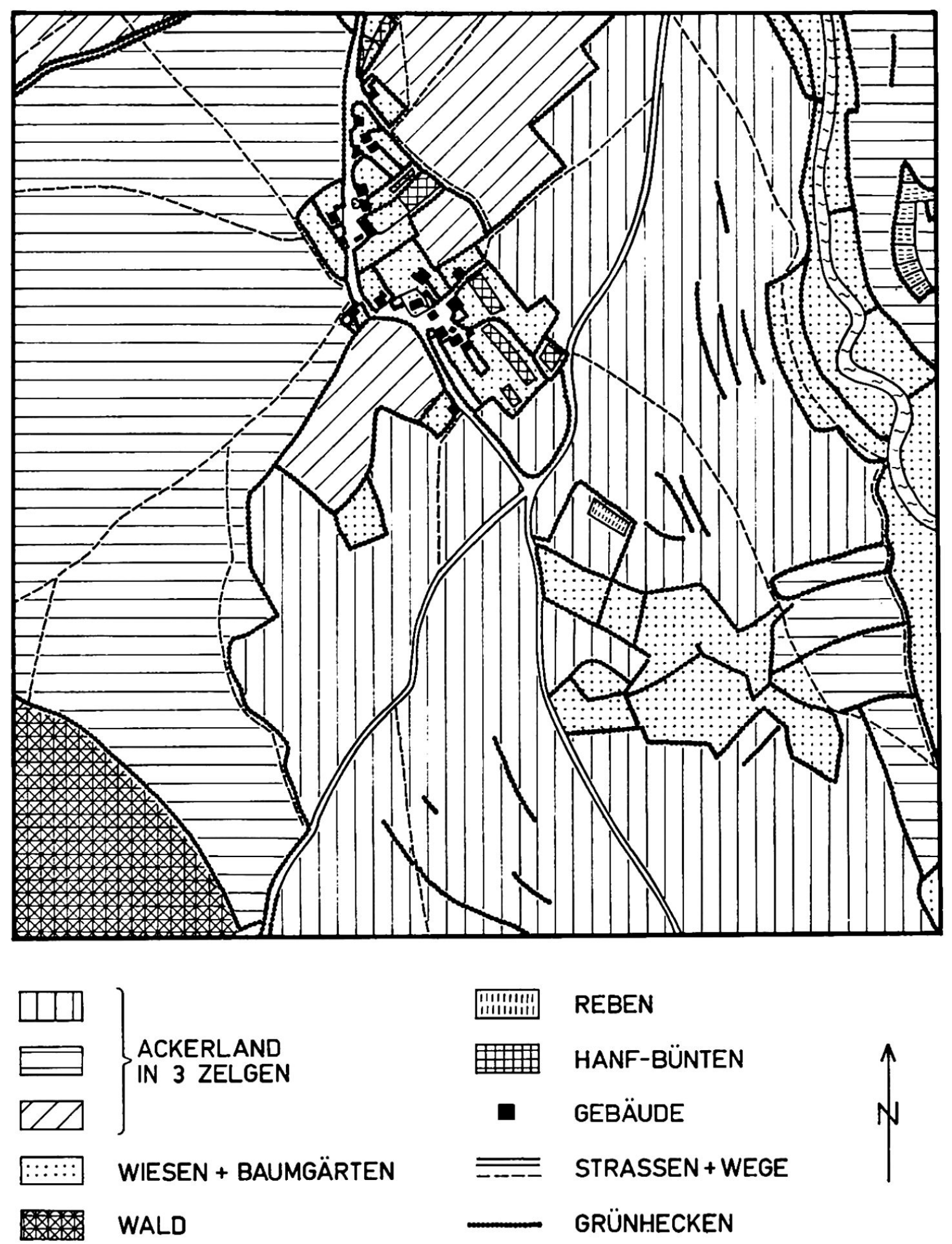

$500 \mathrm{~m}$

Fig. 4. Beispiel für die $\propto$ Halb-Bocage $>$ zur Zeit der Dreizelgenwirtschaft: Umgezeichneter Ausschnitt aus dem Zehntenplan von Dübendorf (Plan Q $32 \mathrm{im}$ Staatsarchiv des Kt. Zürich) von 1681 (Autor: H. J. Hulftegger).

auf die Heckenstandorte eine gewisse Ähnlichkeit mit der eben besprochenen Ajoie. Allerdings ist hier die Auflösung der Bocage im Grünland schon weit fortgeschritten oder ganz abgeschlossen. Reste finden sich etwa noch im Birstal. Dagegen haben sich 
die Hecken auf der Nutzungsgrenze zwischen Kulturland i. e. S. und Weide erhalten (z. B. umhegte Allmenden im Birstal, im Tal von St. Imier, am südlichen Jurafuß). Eine Besonderheit innerhalb der jurassischen Talsiedlungsregionen ist das Hochtal von Les Verrières. Die Dorfflur von Les Bayards bietet ein ganz aussergewöhnliches Bild: Sie ist in eine Reihe von äußerst schmalen Parzellen aufgeteilt, die quer zur Talrichtung verlaufen und sich von einem Hang zum andern bis an die Weidegrenze erstrecken. Der Aspekt der Streifenflur wird physiognomisch in markanter Weise unterstrichen, da auf allen Parzellengrenzen Lesesteinwälle liegen, die mit Gebüsch bewachsen sind (s. Abb. 3). Die Häufigkeit der Sammelsteine läßt sich hier durch das lokale Vorkommen einer skelettreichen Grundmoräne erklären. Die eigenartige Flurform ist wohl auf eine planmäßige Kolonisation des Spätmittelalters (?) zurückzuführen. Im Laufe der Jahrhunderte fand infolge des Erbgangs eine immer weitergehende Teilung statt, und zwar auf der ganzen Länge der Parzellen, sodaß alle gleichermaßen Anteil an gutem und schlechtem Boden hatten. Auf der Flur von Les Verrières sollen früher ähnliche Verhältnisse geherrscht haben (nach einer Mitteilung des Gemeindepräsidiums von Les Bayards und Gutersohn [14]) ${ }^{6}$.

Gelegentlich treten Lesesteinhaufen mit Gebüsch auch in Wiesland auf (Studmatten, vgl. z. B. BURGER [7]).

Auf den Höhen markieren die Hecken entweder die Besitzgrenzen zwischen den meist gut arrondierten Einzelhofarealen oder aber die Nutzungsgrenze zwischen Kulturland i. e. S. und Weide (s. Abb. 4). Im Gegensatz zur vorwiegend vertretenen blockartig aufgeteilten Flur finden wir auch einige wenige Beispiele mit Streifenflur, wobei sich die einzelnen arrondierten Besitzparzellen quer über eine Niederung legen (z. B. Vallée de la Brévine, Mulde von Le Coty, vgl. Gutersohn [14]). In einigen Fällen kommen in Höhenlagen auch Komplexe von Grünland vor, die durch Hecken in Privatparzellen abgeteilt sind und von Dörfern aus bewirtschaftet werden. Bekannt sind uns in dieser Hinsicht der Mont Aubert (Gutersohn [14]) und der LägernNordhang. Es handelt sich dabei wohl um die Aufteilung eines dörflichen Allmendstückes und die Umwandlung in privates Wiesland, da der Ertrag des dorfnahen Heulandes nicht ausreichte. In neuerer Zeit werden die Parzellen allerdings zum Teil wieder als Weide genutzt. Wir haben noch darauf hinzuweisen, daß die Kulturhecken, die den östlichen Teil des Kettenjuras einnehmen (etwa östlich einer Linie SolothurnDelsberg), im Westen durch Trockenmauern mit analogen Funktionen abgelöst werden (etwa westlich der Linie Biel-St. Ursanne). Dazwischen liegt ein Übergangsgebiet. Auf diesen Mauern wächst aber oft wildes Gebüsch, sodaß sich mitunter ein dem östlichen Kettenjura konvergentes Landschaftsbild ergibt.

4) Freiberge: Wir haben das Gebiet der Freiberge vom übrigen Kettenjura abgetrennt und ihm in der Karte (Fig. 2) die gleiche Signatur wie dem angrenzenden französischen Plateaujura gegeben, da gewisse übereinstimmende Merkmale inbezug auf die Siedlungs- und Flurform festzustellen sind. Infolge des weniger akzentuierten Reliefs haben wir es hier mit einer mehr oder weniger innigen Vermischung, nicht mit einer Trennung von geschlossener und Streusiedlung zu tun. Jenachdem sind die Fluren gut oder mäßig arrondiert. Besitz- und Nutzgrenzen sind durch Trockenmauern mit oft starkem Bewuchs (Wildhecken) markiert. Im Bereich der Dorfsiedlungen hat früher die Dreizelgenwirtschaft geherrscht (LEU [28]). Die einstige starke Ausdehnung des Ackerlandes in Form großer offener Komplexe wird durch Flurnamen wie «Fin» (Zelg), «Longs Champs», «Grands Champs» und dgl. bezeugt ${ }^{7}$. Vom damaligen Landschaftsbild ist nicht mehr viel übrig geblieben. Der Übergang zur Viehwirtschaft und die zum Teil relativ spät entstandene Streusiedlung haben zu einer

6 Ähnliche Flurformen mit Hecken auf Lesesteinwällen finden sich auch in Teilen des benachbarten französischen Juras, so bei Longeville Haute und Métabief. Allerdings sind dort die Parzellen nicht so extrem schmalstreifig.

7 Derartige Flurnamen finden sich in ähnlicher Weise im französischen Plateaujura. 


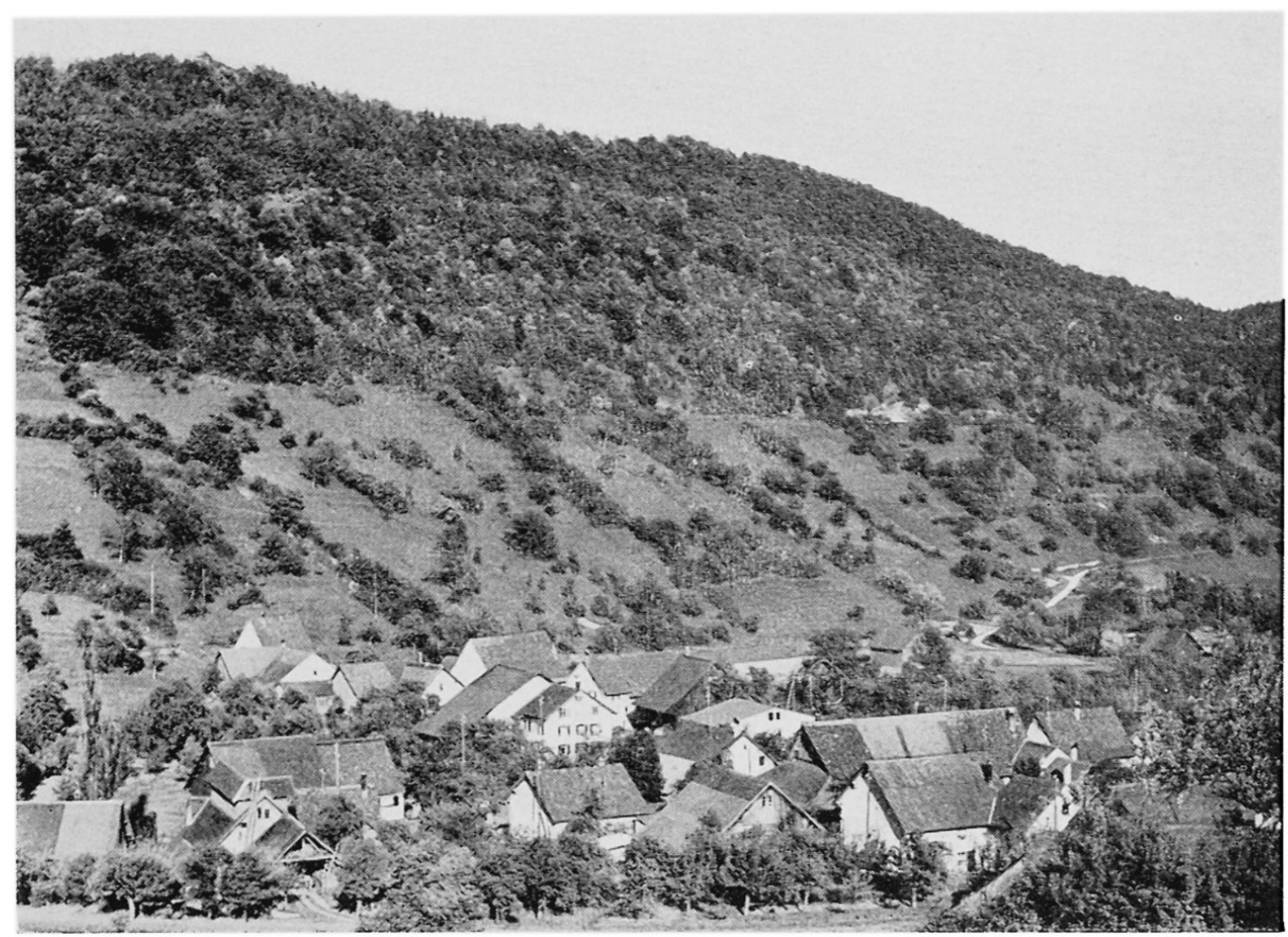

Abb. 1. Blick auf den steilen Hang der Gräte bei Merishausen (SH). An Stelle der einstigen Rebberge (vgl. Erstausgabe des Siegfried-Atlas von 1883, Blatt 12, Merishausen) finden wir heute nur noch die vorwiegend mit dem Gefälle verlaufenden und mit Wildhecken bewachsenen Lesesteinriegel und Magerwiesennutzung. Aufnahme D. Steiner 25. 5. 1958.

umwälzenden Veränderung der Nutzungs- und Siedlungsstruktur geführt. Immerhin finden wir auch heute noch in der Nähe der Dörfer das von Einhegungen freie Kulturland i. e. S. («Finages»). Es darf auch vermutet werden, daß längere Lesesteinriegel mit Wildhecken in Grünland (z. B. Studmatten bei Les Breuleux) darauf hinweisen, daß diese Flächen früher einmal unter Pflug standen.

5) Mittelland (ohne Umgebung von Genf): Wir rechnen dieser Zone die Gebiete der Dorfsiedlungen zu und grenzen sie gegen eine subalpine Zone ab, in der die Streusiedlung dominiert. Für ihren ganzen Bereich ist die einstige Dreizelgenwirtschaft nachgewiesen (vgl. z. B. Volkart (45), Chevallaz [8]). Die früher zahlreich vertretenen Hecken (vgl. letzter Abschnitt) sind bis auf wenige Reste verschwunden. Ein großer Teil ist zweifellos noch dem Mehranbau während des letzten Krieges zum Opfer gefallen. Erhalten geblieben sind in erster Linie Wildhecken auf Ackerrainen, wo sie relativ wenig stören. In besonderem Maße ist dies noch im westlichen Mittelland (Kt. Waadt und Fribourg) der Fall, wo dieser Heckentypus noch recht häufig vertreten ist. Auch hier werden die Gebüsche aber im Laufe von Güterzusammenlegungen gerodet, wie etwa das Beispiel von Grandcour (VD) zeigt.

6) Kt. Gcnf: Wir haben dieses Gebiet vom übrigen Mittelland als besonderen Fall abgetrennt, da hier die Kulturhecken stark zunehmen. Man kann diese Erscheinung als Ausläufer der angrenzenden Bocage auf französischem Boden betrachten (man beachte die Zunahme der Heckendichte auf Fig. 1 mit dem Überschreiten der Landesgrenze). Das heutige Landschaftsbild zeigt ein Vorwiegen der Dorfsiedlungen und ein Mosaik von offenen und mehr oder weniger geschlossenen Parzellenblöcken. In Analogie zur Entwicklung im Pays de Gex jenseits der Grenze (s. Lebeau [26]) darf 


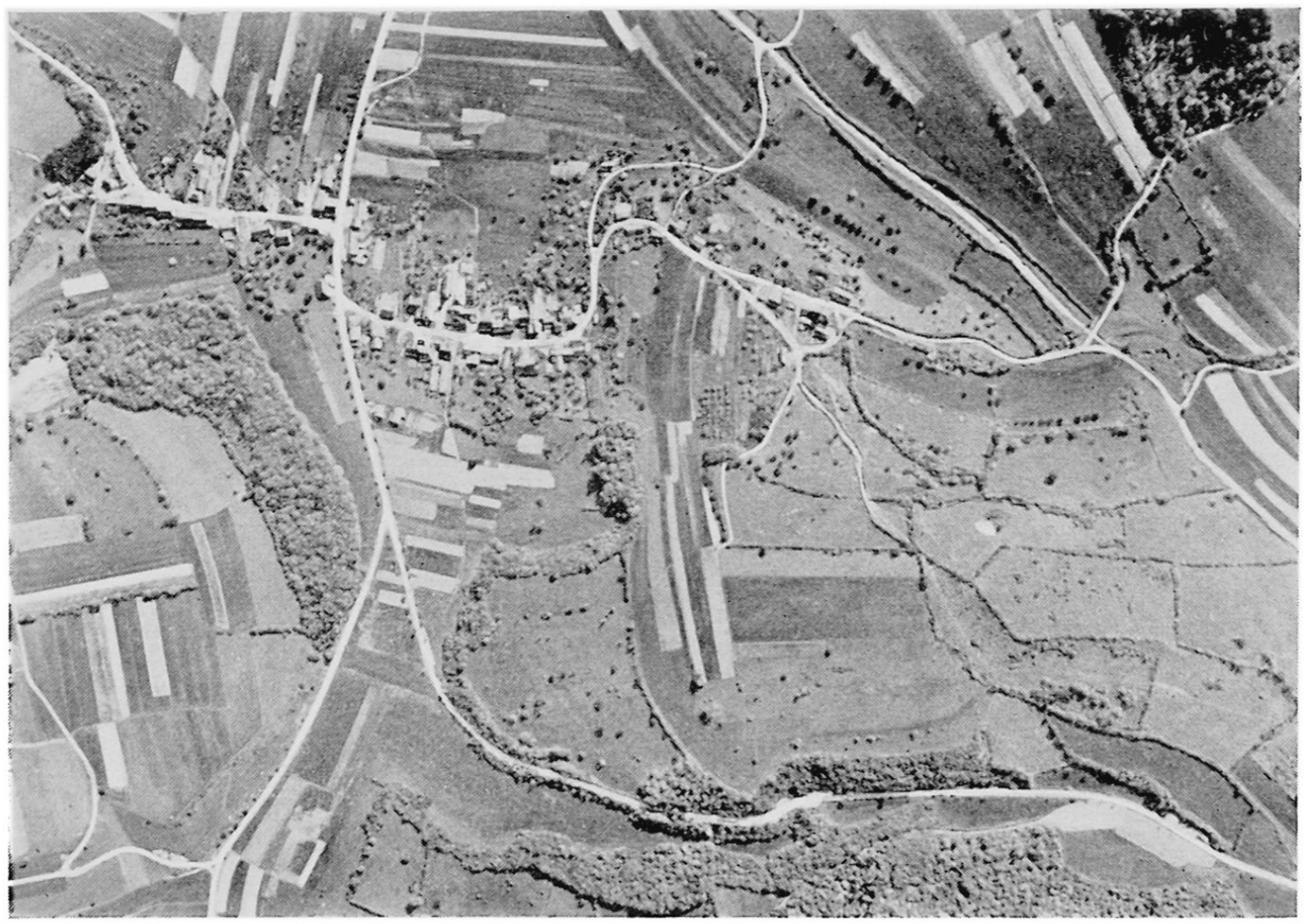

Abb. 2. Luftbildausschnitt aus der heute noch bestehenden "Halb-Bocage * bei Rocourt (Ajoie). Eingehegte Grünlandparzellen wechseln mit offenen Komplexen (vorwiegend Ackerland) ab. Schräg durch die rechte obere Ecke zieht sich der Rest einer von Hecken begleiteten Viehtrift.

Bildlänge ca. 1,6 km, Nord am linken Bildrand. Aufnahme: Eidg. Landestopographie 16. 5. 1936

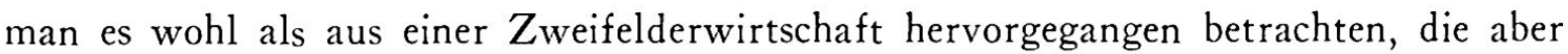
keinen Flurzwang kannte ${ }^{8}$. Dies begünstigte die Entstehung von aus privaten Grünlandparzellen zusammengesetzten Bocage-Komplexen. Dazu war allerdings die Befreiung vom Servitut der Gemeinatzung notwendig, die auch in diesem Gebiet üblich war. Das Bestehen dieses Brauches gab aber der Einhegung dieser Wiesenflächen erst ihren Sinn. Wenn sich, trotz dem Fehlen des Flurzwanges, auch hier eine recht deutliche Trennung in offene (Ackerland) und geschlossene Flächen (Wiesen) durchsetzen konnte, so ist dies zweifellos in erster Linie auf naturgeographische Gegebenheiten zurückzuführen. Die heutige Nutzungsstruktur stimmt damit allerdings nicht mehr überein: Die vermehrte Ausrichtung auf den Ackerbau hatte zur Folge, daß auch Parzellen innerhalb der Bocage-Komplexe unter Pflug genommen wurden.

7) Subalpine Zone: Eine fast durchgehende subalpine Zone mit einzelnen ausgesprochenen Zentren (vgl. Fig. 1) ist das Verbreitungsgebiet von Kulturhecken, die mit der hier vorherrschenden Streusiedlung und der im allgemeinen guten Arrondierung der Wirtschaftsflächen verknüpft sind. Die Hecken stehen fast alle ohne Ausnahme auf den Besitzgrenzen. Allerdings ist das Netz nicht mehr vollständig, sondern nur noch recht lückenhaft (s. Fig. 3). Der heutige Zustand und die herrschende Praxis der Bauern lassen erkennen, daß auch hier die Auflösung stark im Fortschreiten begriffen ist: Entstandene Lücken werden nicht durch die Neuanpflanzung von Sträuchern ausgebessert, sondern lediglich durch Drahtzäune verstärkt, sofern solche zur Einfassung von Mähweiden nötig sind. Das allmähliche Verschwinden der Hecken erklärt sich aus

8 Dafür spricht auch, daß eine frühere Zweifelderwirtschaft im Gebiet südlich von Nyon nachgewiesen werden konnte (CHevallaz [8]), das somit offenbar den letzten Ausläufer dieser alten Wirtschaftsform im westschweizerischen Mittelland darstellt. 


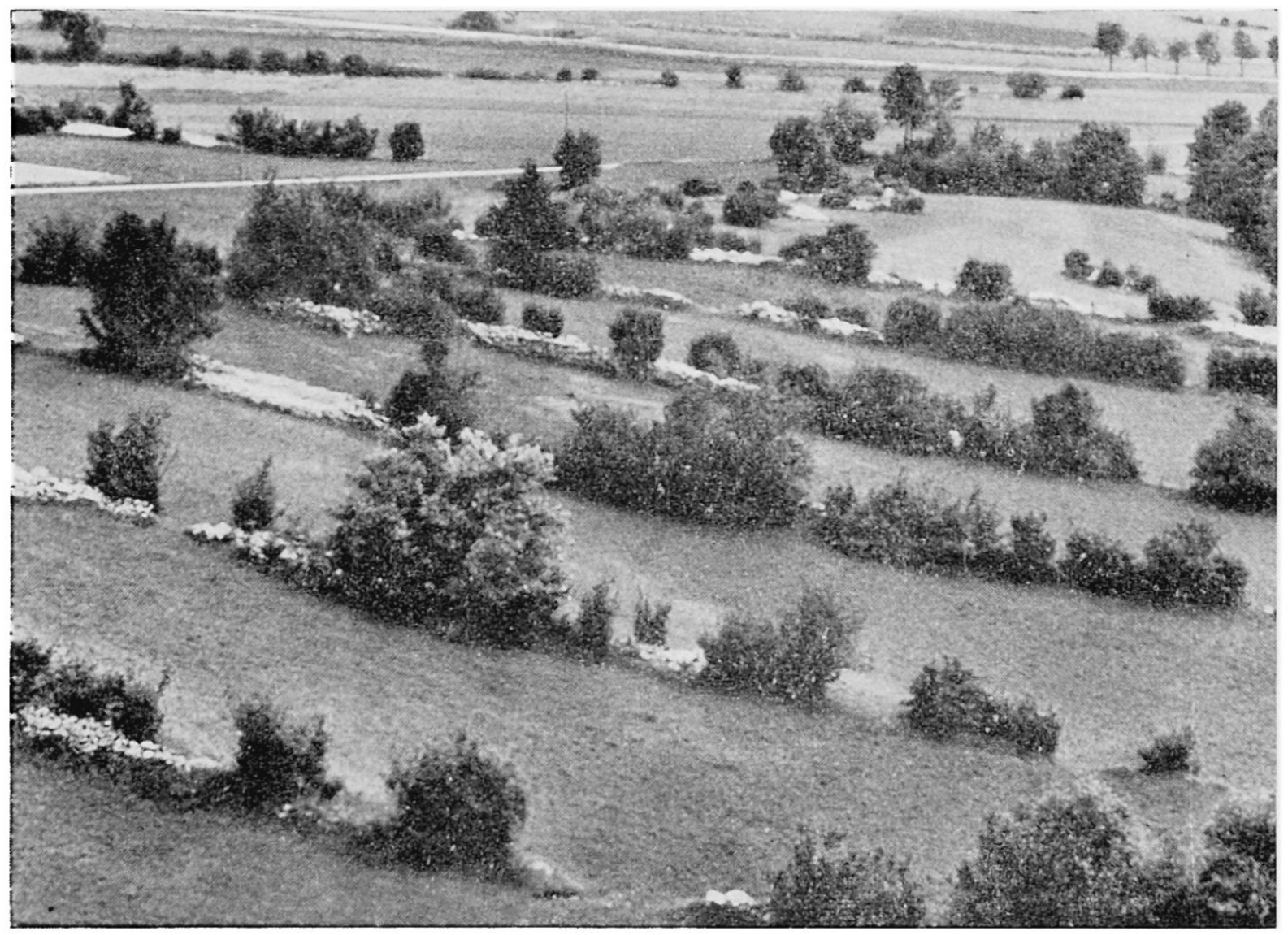

Abb. 3. Ausschnitt aus der auffallenden Schmalstreifen-Flur von Les Bayards (NE). Die Parzellengrenzen werden durch Lesesteinwälle markiert, die zu einem guten Teil von Gebüsch überwachsen sind. Aufnahme: W. Nägeli 1947.

dem weitgehenden Funktionsverlust. In früherer Zeit herrschte in dieser Zone eine extensive Form der Feldgraswirtschaft, wobei die Fruchtfolge der Ackerkulturen durch eine mehrjährige Periode der Weidenutzung abgelöst wurde (vgl. z. B. Pulver (34) für das Emmental, Pfyffer (33) für den Kt. Luzern). Zu jener Zeit hatten die Hecken somit nicht nur die Funktion der damals noch wichtigen augenfälligen Besitzgrenzmarkierung, sondern auch die Aufgabe, das weidende Vieh am Überlaufen zu hindern. Außerdem nahm das Einhegungsnetz zum Teil nicht nur die Besitzgrenzen ein, sondern verdichtete sich innerhalb der arrondierten Areale zur Unterteilung der Fläche in einzelne Schläge. Dies war im Verbreitungsgebiet der geregelten Feldgraswirtschaft der Fall, bei der in geordnetem Turnus Acker- und Weidenutzung schlagweise miteinander abwechselten (PFYFFER [33]) und die mit der alten holsteinischen Koppelwirtschaft (s. MARQUARDT [29]) verglichen werden kann. Im Gegensatz zur geregelten erfolgte der Nutzungswechsel bei der wilden Feldgraswirtschaft nicht nach einem bestimmten Schema. Die jeweilen aus dem Weideland neu umgebrochene Ackerfläche wurde für die Dauer des Anbaus durch einen transportablen Holzzaun geschützt. Anstelle dieser alten Wirtschaftsformen finden wir heute moderne Varianten der Feldgraswirtschaft (Graswirtschaft mit etwas Ackerbau, Kleegraswirtschaft) oder reine Graswirtschaft. Das in neuerer Zeit eingeführte Prinzip der Mähweide konnte den Auflösungsprozeß der Hecken nicht aufhalten, da dafür leicht verschiebbare Elektrozäune bevorzugt werden.

In den Gebieten mit bereits größeren Höhenunterschieden und steilerem Gelände (z. B. Emmental), sind auch, ähnlich wie im Kettenjura, Dauerweideflächen ausgeschieden und durch Hecken vom Kulturland i. e. S. getrennt. 


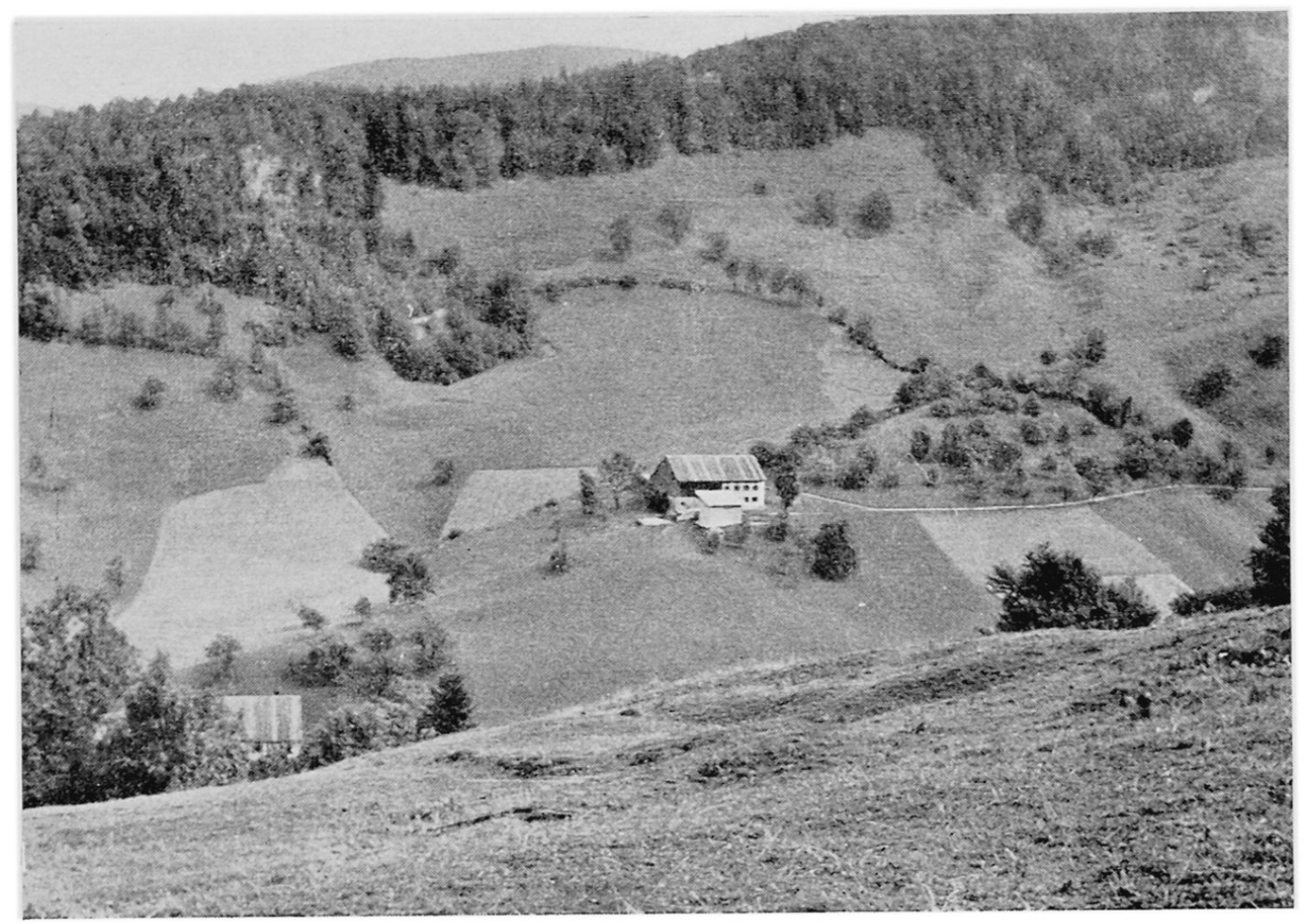

Abb. 4. Typisches Bild aus dem östlichen Kettenjura: Der Hof "Schilt " nördlich des Paßwang. Einerseits ist das arrondierte Hofareal gegenüber den Nachbarhöfen und anderseits das Kulturland i. e. S. gegen die Dauerweide durch - allerdings lückenhafte - Kulturhecken abgegrenzt.

Aufnahme: D. Steiner 31.8.1958

8) Randalpine Täler: Ohne markante Grenze geht der subalpine Saum in die Zone der randalpinen Hecken über. Der wesentlichste Unterschied gegenüber Zone 7 besteht darin, daß hier die typisch alpine Aufteilung des Wirtschaftsareals in Nutzungsstufen einsetzt. Die vertikalen Distanzen werden so groß, daß außerhalb der Wirtschaftszentren Außenställe unterhalten werden. Außerdem haben wir es wieder vermehrt mit Dorfsiedlung und damit mit Parzellen in Gemengelage zu tun. Die Hecken treten in den untern Teilen der Talhänge als Abgrenzung von mehr oder weniger blockförmigen Wiesenparzellen auf, in denen die Außenställe stehen (s. das Beispiel des Simmentals in Abb. 5). Häufig greift diese Nutzungszone auch auf Schuttkegel über. Auf den Parzellengrenzen sind dann Lesesteinhaufen oder Mäuerchen aufgeschichtet, die wohl vor der Zeit der Verbauungen ständigen Zuwachs durch die Abräumung der Wiesen nach Wildwasserüberschwemmungen erhielten und die heute von Wildhecken überwachsen sind (z. B. Kt. Glarus ${ }^{9}$, Churer Rheintal). Oft sind auch größere Weideareale (etwa Churer Rheintal) oder auch kleinere Heimweiden (z. B. am südlichen Zürichseeufer im Kt. Schwyz) durch Hecken vom Kulturland i. e. S. getrennt. Wie weit auch hier die historischen Veränderungen (insbesondere die Umwendung von der Selbstversorgung zur spezialisierten Graswirtschaft) für die Entstehung oder das Verschwinden von Hecken eine Rolle gespielt haben, wäre abzuklären. Die heutigen Tendenzen zielen im allgemeinen auf eine Rodung zumindest der gepflanzten Hecken (z. B. im Kt. Glarus ${ }^{10}$ ).

9) Inneralpine Täler: In dieser Zone finden wir geschlossene Siedlungen und, begünstigt durch das trockene Klima, einen starken Anteil des Ackerbaues am Kulturland

9 Mündliche Mitteilung von J. Hösli.

10 Mündliche Mitteilung von E. Landolt. 


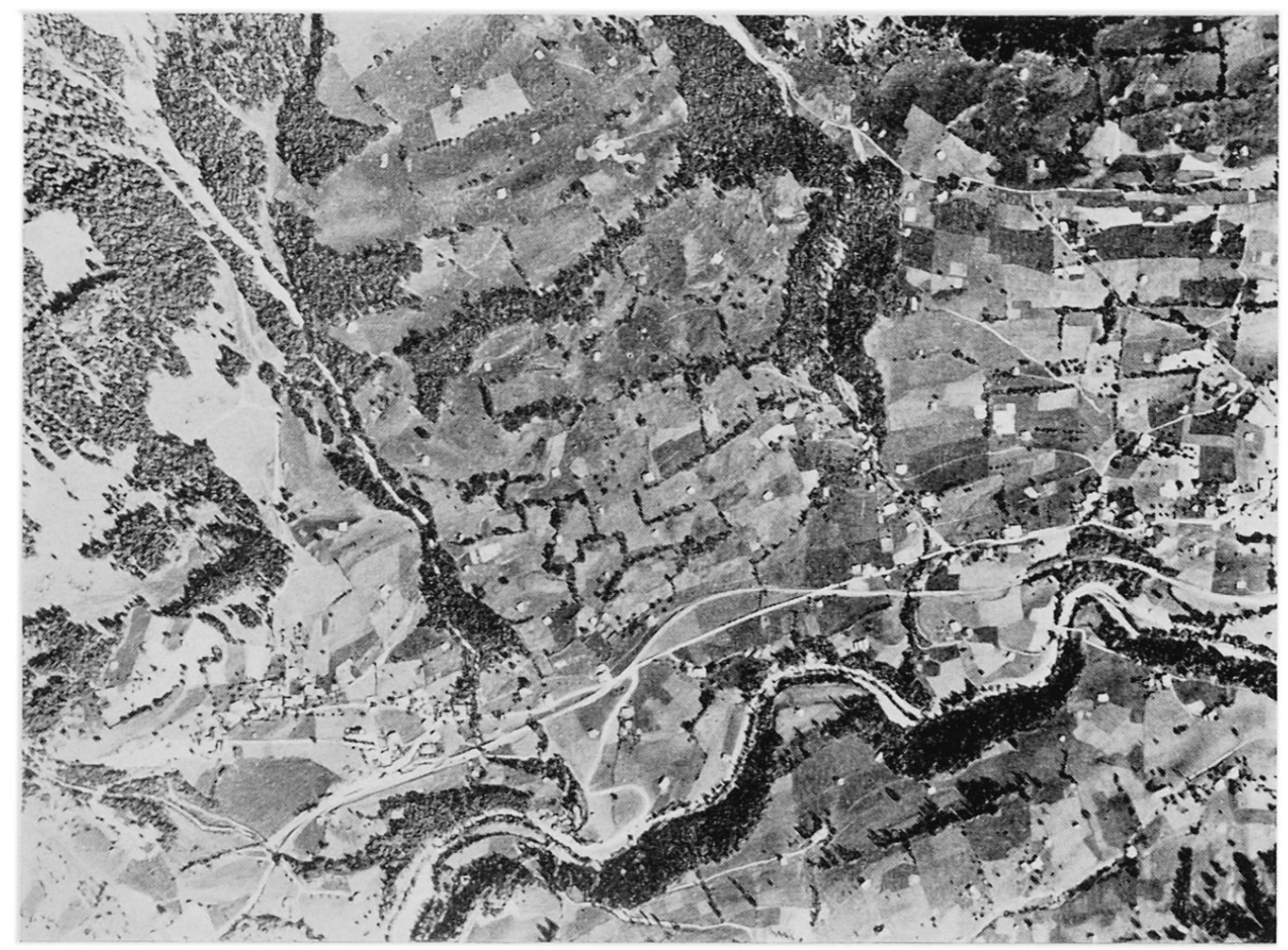

Abb. 5. Luftaufnahme vom Niedersimmental bei Oberwil. Hecken umrahmen einen großen Teil der von Außenställen aus bewirtschafteten Wiesenparzellen an den Talhängen. Bildlänge ca. 2,5 km, Nord am obern Bildrand. Aufnahme: Eidg. Landestopographie 6.9.1940

i. e. S. Dieser nutzt günstige Expositionen aus und nimmt große Teile des ackerfähigen Bodens an den Talhängen ein. Die Nutzung steiler Hänge setzt aber eine Terrassierung voraus, wobei oft die einzelnen Stufenkanten durch aufgeschichtete Steine verstärkt werden. Mäuerchen, Lesesteinriegel und Ackerraine bilden somit hier wiederum geeignete Standorte für das Aufkommen von Wildhecken, die denn auch häufig den terrassierten Hängen ihr charakteristisches Gepräge verleihen (vgl. Abb. 6). Gebüschstreifen wachsen aber auch auf ähnlich beschaffenen Stellen im Wiesland, so etwa auf Hangknicken, die durch die Anlage von Wegen entstanden sind, oder entlang von mit Steinen eingefaßten Bewässerungsgräben (Wallis). In einzelnen Fällen sind in Grünland auf Schuttkegeln auch geschlossene Heckennetze auf Lesesteinwällen entstanden (auffallendstes Beispiel: Ill-Schuttkegel bei Suste/VS).

10) Tessiner Hügelland: Hier sind die Hecken, wie überhaupt im Tessin, recht selten. Sie dürften etwa als wilde Gebüsche auf Mäuerchen vorkommen. Gelegentlich finden sich Hecken zur Abgrenzung größerer Weideflächen in höheren Lagen.

\section{Die Genese der Kulturhecken und ihr Standort in der historischen Wirtschaftsland- schaft}

Aus dem bisher gesagten ergibt sich, da $B$ heute noch bestehende gepflanzte Hecken mitunter recht aufschlußreich für die Rekonstruktion früherer wirtschaftlicher Zustände sein können. Insbesondere wollen wir im folgenden Entstehung und Bedeutung der Hecken im Bereich der einstigen meist zelgengebundenen Felderwirtschaften etwas näher beleuchten.

Aus alten Karten und Chroniken (z. B. Karte des Kt. Zürich von Gyger (54), Chronik von StumpF, s. WinkLer [5o]) geht hervor, daß z. B. das Mittelland in früherer Zeit äußerst hekkenreich gewesen sein muß. Auf den ersten Blick widerspricht dies dem Charakter der im gleichen Gebiet damals verbreiteten kollektiven Wirtschaftsform mit Flurzwang und Gemeinatzung. 


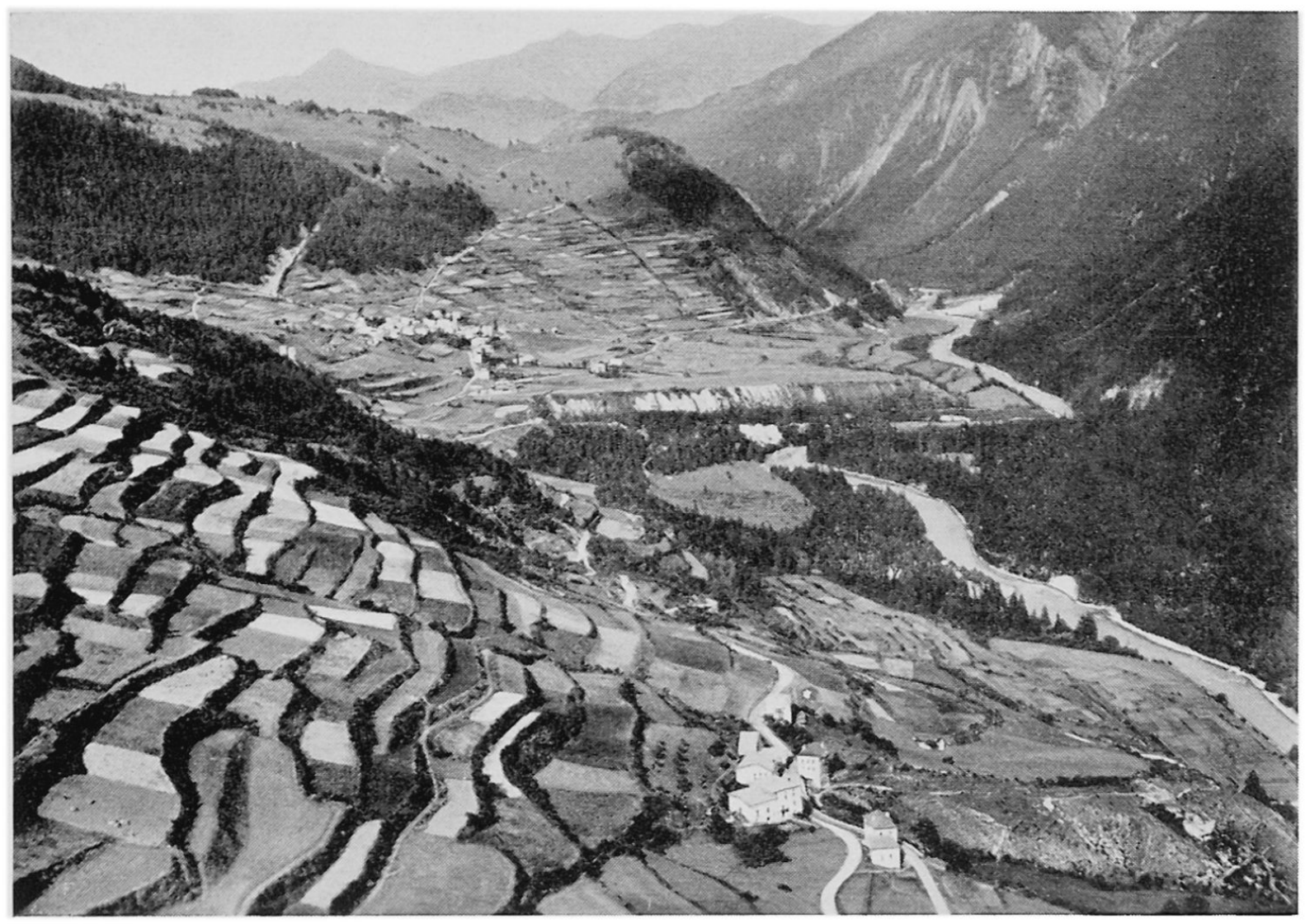

Abb. 6. Die Gegend von Ramosch (Unterengadin) als Beispiel für die terrassierten Hänge der inneralpinen Täler. Die Photo zeigt, wie die Raine zwischen den Parzellen häufig von wildem Gebüsch bewachsen sind. Aufnahme: Swissair-Photo AG 3.9.1947

Es ist das Verdienst von Lebeau 26), am Beispiel des französischen Südjura gezeigt zu haben, $\mathrm{da} \beta$ das eine das andere nicht ausschließen muß. Die bisherige Literatur über Heckenlandschaften ist vielleicht etwas schuld daran, $d a \beta$ man im allgemeinen den Gegensatz zwischen Bocage und «Openfield» mit räumlich getrennten Regionen verschiedener Siedlungs- oder Wirtschaftsformen in Zusammenhang bringt. Es ist aber eben nicht zu vergessen, daß sie sich vorwiegend mit dem maritimen Grünlandgürtel Westeuropas befaßt, in dem die Entwicklung der Agrarlandschaft andere Wege als in Zentraleuropa gegangen ist. Auf alle Fälle müssen wir für das Verbreitungsgebiet der alten Felderwirtschaften in der Schweiz ein Nebeneinander von eingehegten und offenen Komplexen annehmen. Dies ergibt sich aus verschiedenen Quellen (s. unten). Die eingehegten Parzellen dokumentieren die Notwendigkeit des Anbaus von Kulturen, die sich nicht in die normale Rotation einordnen lassen (z. B. Hanf und Flachs) oder das Bedürfnis nach individueller ertragssteigernder Nutzung (z. B. Wiesen). Die Hecken machen dabei einerseits den privaten Besitzanspruch deutlich (vgl. BADER [4]) und schützen anderseits vor dem Vieh auf der Gemeinweide. Die Bocage-Parzellen sind wohl zum größten Teil durch eine partielle - rechtmäßige oder unrechtmäßige - Aufteilung der kollektivwirtschaftlich genutzten Fläche hervorgegangen. Der Gegensatz zwischen Bocage und Openfield spiegelt somit in diesem Fall den Streit zwischen individueller und gemeinwirtschaftlicher Nutzung innerbalb desselben Wirtschaftsraumes wieder. Sicher hat dabei auch eine gewisse Anlehnung an natürliche Voraussetzungen eine Rolle gespielt, indem die Aufteilung am ehesten zuerst auf Stellen einsetzte die für den Ackerbau ungünstig waren. Der Streit erstreckte sich über Jahrhunderte und somit ist die etwa auf einem alten Flurplan dargestellte «Halb-Bocage» nur die Momentaufnahme eines dynamischen Zustandes. Die Konkurrenz zwischen den zwei betrieblichen Prinzipien wurde erst mit der Aufhebung von Flurzwang und Gemeinatzung ihres Sinnes beraubt und fand damit ihren Abschluß.

Wir geben nun anhand verschiedener Quellen (Flurpläne im Staatsarchiv des Kt. Zürich (58), Haupturbar der Grafschaft Lenzburg (59) 11, Angaben aus der Literatur wie z.B. GaLLUSSER [11]) eine Übersicht über die möglichen Standorte von Hecken in der Dreizelgenwirt-

11 Die Angaben aus diesem Urbar verdanken wir P. SteIner. Zusammen mit dem in seiner Arbeit (39) rekonstruierten Flurplan des Gerichtes Reinach (AG) vermittelten sie uns wertvolle Anhaltspunkte. 
schaft (vgl. dazu Fig. 4). Sie können folgende Nutzungs- oder Wirtschaftsflächen einhegen: 1) Die Hofstätten der dörflichen Siedlung mit den Baum- und Gemüsegärten. In diesem Fall bilden die Hecken in ihrer Gesamtheit gegen außen den Dorfetter, der zwar in der Literatur meistens als Holzzaun angegeben ist, der aber, wie sich aus den Flurplänen ersehen läßt, recht häufig einen Grünhag darstellte. 2) Ackerbaulich genutzte Privatparzellen außerhalb der Hofstätten, wie z. B. die sog. Bünten (Anbau von Hanf und Flachs) oder Einschläge innerhalb der Allmend. 3) Das Wiesland als Ganzes und die einzelnen Parzellen im besonderen. Es sind vor allem diese Grünlandkomplexe, die lokal einen Bocage-Aspekt erzeugten. 4) Die Ackerzelgen als Ganzes gegen die übrigen Nutzungsflächen. Oft sind z. B. die vom Dorf wegführenden und als Viehtriften verwendeten Straßen beidseits von Hecken begleitet («Gassen»). Innerhalb der Zelgen kommen nur wilde Gebüschstreifen auf Lesesteinhaufen oder Rainen vor 12. 5) Die Allmendweide. 6) Die Wirtschaftsareale von Einzel- und Sonderhöfen, die nicht dem dörflichen Wirtschaftssystem angehören. 7) Die Dorfgemarkung als Ganzes.

Zusammenfassend können wir sagen, da $\beta$ das Verbreitungsgebiet der Felderwirtschaften in historischer Zeit von einer Halb-Bocage eingenommen wurde, die heute bis auf wenige Reste (Ajoie, Kt. Genf) vollkommen aufgelöst ist. Im Gegensatz dazu läßt sich der Bereich der jurassischen und subalpinen Feldgraswirtschaften als Voll-Bocage charakterisieren, die in stark rudimentärer Form noch erhalten ist.

\section{Der Aussagewert der Hecken für die geographische Forschung}

Die Bedeutung der Hecken für die kulturlandschaftsgeschichtliche Forschung ergibt sich aus den vorhergehenden Abschnitten. Wir möchten die wichtigsten Punkte hier nochmals zusammenstellen und auch einige weitere, bisher nicht betrachtete Aspekte berücksichtigen, die für andere Arbeitsrichtungen von Wichtigkeit sein können.

Bei der Untersuchung der Naturlandschaft können Hecken in folgender Weise von Nutzen sein: 1) Wildhecken können als Vorstufe zu einer Wiederbewaldung aufgefaßt werden und sagen deshalb etwas über die potentielle natürliche Vegetation aus. Es ist allerdings zu beachten, daß die dafür in Frage kommenden Stellen innerhalb des untersuchten Gebietes nicht normale, sondern extreme (trockene) Standorte darstellen. Wir sind auf die pflanzensoziologische Stellung der Wildhecken in der Schweiz nicht eingetreten und verweisen auf die zuständige Literatur (z. B. Tüxen (42), GAMs (12), Braun-Blanquet (5), Wilczek [48]). 2) Die regionale Verbreitung von Lesesteinhaufen, aspektmäßig verstärkt durch darauf wachsendes Gebüsch, sagt unter Umständen etwas über die Ausdehnung einer bestimmten geologischen Formation im Untergrund aus. 3) In sedimentären Gebieten betonen gelegentlich heckenartige Gebüschstreifen auf Anstehendem das geologische Streichen (z. B. auf dem Schänerberg nördlich des Walensees).

Für die Kartierung und Typisierung der heutigen Kulturlandschaft können Hekken in verschiedenen Richtungen verwendet werden ${ }^{13}: 1$ ) Als spezielle, besonders auffallende Variante der möglichen Einzäunungsarten kann die Grünhecke ein Kriterium zur Abgrenzung von Agrarlandschaften abgeben (vgl. WindLeR [49]). 2) Als funktionales (Kulturhecken) oder auch lediglich formales Element (Wildhecken) auf Parzellengrenzen unterstreichen die Hecken die Art der Flurformen. 3) Als Schutzhecken an der Grenze von Weideflächen erleichtern sie die Ausscheidung verschiedener Nutzflächen (vgl. VöGELI [44]). 4) In Streusiedlungsgebieten können mit Hecken auf Besitzgrenzen einzelne Wirtschaftsareale voneinander unterschieden werden. 5) In den inneralpinen Tälern läßt sich mit der Verbreitung der Terrassierungen an den Hängen, die durch Wildhecken unterstrichen werden, das Dauerackerland im Falle

12 Stufen treten auch auf mehr oder weniger flachem Gelände als Zelggrenzen oder Umrahmung von Zelgteilen auf. Für ihre Entstehung werden verschiedene Möglichkeiten in Betracht gezogen: Humussedimentation unter erst später gerodetem Wald (GROSJEAN [13]; Vermehrte Abspülung im Freiland gegenüber einer erst später gerodeten Fläche (SchmiтhüsEN [36]); Pflugtätigkeit (Gallusser [11]). Solchen anthropogenen Kleinformen muß stärkere Beachtung geschenkt werden.

13 Vor allem für diese Fälle sei betont, daß das Luftbild, das bei genügend großem Maßstab alle Hecken deutlich zeigt, als zeitsparendes Hilfsmittel gute Dienste leisten kann. 
von Felderwirtschaften und die periodisch unter Pflug stehende Fläche im Falle von Feldgraswirtschaften erfassen.

Für die Interpretation der kulturlandschaftlichen Genese haben die Hecken folgenden Aussagewert: 1) Die Kenntnisse über den Standort der Kulturhecken in der historischen Wirtschaftslandschaft hilft bei der Rekonstruktion der alten Zustände (Wirtschaftsareale, Nutzflächen, betriebliche Systeme). 2) Wildhecken auf Lesesteinriegeln und Ackerraine in Grünland lassen eine früher größere Ausdehnung der bebauten Fläche erkennen.

Der vorgelegte Beitrag erhebt keinen Anspruch auf Vollständigkeit, sondern ist nur als erste Orientierung über ein wenig bekanntes Thema aufzufassen. Viele der angetönten Probleme müssen im Detail weiter verfolgt werden.

\section{Literatur}

\section{BENÜTZTE QUELLEN}

1) Amberg, KarL: Der Pilatus in seinen pflanzengeographischen und wirtschaftlichen Verhältnissen. Luzern 1916. 2) AuBert, SAM.: La lisière et la haie. Revue Forestière Suisse 84: 73-76, Bern 1933. 3) Aubert de la Rūe, E.: Observations géographiques et ethnographiques sur les clôtures rurales. La Nature 78: 1-8, Paris 1950. 4) BADER, KARL S.: Gartenrecht. Zeitschr. der Savigny-Stiftung für Rechtsgeschichte 75: 252-273, Weimar 1958. 5) Braun-BlanQueT, J.: Übersicht über die Pflanzengesellschaften Rätiens. Vegetatio, Acta Geobotanica 2 (4/5): 341-360, Den Haag 1949. 6) BRoDBECK, Chr.: Landwirtschaft und Meliorationen. Natur und Landschaft Heft 4, Bașel 1957. 7) BuRGER, Hans: Wytweiden und Studmatten. Schweiz. Zeitschr. für Forstwesen 78: 366-369, 1927. 8) Chevallaz, Georges André: Aspects de l'agriculture vaudoise à la fin de l'ancien régime. Diss. Univ. Lausanne 1949. 9) Davis, D. G.: Zum Problem der Heckenlandschaft. Erdkunde 7 (2): 139-140, Bonn 1953. 10) Escher, W.: Zäune - Mauern - Hecken. Karte I 73 des Atlas der Schweiz. Volkskunde, herausg. von Paul Geiger und Richard Weiss, Erlenbach 1958. 11) Gallusser, WeRNER A.: Die Dreizelgenflur im Laufener Jura und ihre heutige Verbreitung im Nahbereich von Basel. Regio Basiliensis 1 (1): 3-10, 1959. 12) Gams, Helmut: Von den Follatères zur Dent de Morcles. Beitr. zur Geobotan. Landesaufn. Heft 15, Bern 1927. 13) Grosjean, Georges: Die Flur von Treiten und ihre historische Aussage. Archiv des Histor. Vereins des Kt. Bern 44 (2): 317-338, 1958. 14) Gutersohn, Heinrich: Geographie der Schweiz Bd. 1: Jura. Bern 1958. 15) Hager, KarL: Verbreitung der wildwachsenden Holzarten im Vorderrheintal. Erheb. über die Verbr. der wildw. Holzarten in der Schweiz, Lfg. 3, Bern 1916. 16) HaRTKE, WolfGang: Die Heckenlandschaft. Erdkunde 5 (2): 132-152, Bonn 1951. 17) Höhn-Ochsner, Walter: Die Pflanzen- und Tierwelt unserer Heimat. 8. Neujahrsblatt der Leseges. Wädenswil für 1937. 18) HörLER, MARTIN : Betriebswirtschaftliche Untersuchungen im Appenzellerland. Sek. Lehrer-Schlußarbeit (Manuskr.), Geogr. Inst. Univ. Zürich 1955. 19) Huffel, G.: La Haye. Etude de sémantique, de géographie et d'histoire forestière. Revue des Eaux et Forêts 71: 757-769 und 849-860, Paris 1933. 20) JEssen, O.: Heckenlandschaften im nordwestlichen Europa. Mitt. der Geogr. Ges. Hamburg 45: 11-58, 1937. 21) Jessen, O.: Feldeinfriedigungen im Bild der Kulturlandschaft. Geogr. Zeitschr. 43: 136-144, 1937. 22) Juil.taRD, E. und MeYnier, A.: Die Agrarlandschaft in Frankreich. Übersetzt von W. HarTKe. Münchner Geogr. Hefte 9, 1955. 23) KläuI, P. und Imhof, Ed.: Atlas zur Geschichte des Kantons Zürich. Zürich 1951. 24) Kunn, WolfGang: Hecken, Terrassen und Bodenzerstörung im Hohen Vogelsberg. RheinMainische Forsch. Heft 39, Frankfurt a/M 1953. 25) LaUtensach, Hermann: Feldheckenstudien in Westmecklenburg. Peterm. Geogr. Mitt. 94: 70-82, 1950. 26) Lebeau, René: La vie rurale dans les montagnes du Jura méridional. Inst. des Etudes Rhodan. de l'Univ. de Lyon, Mémoires et Documents No. 9, 1955. 27) Lebon, J. H. G.: The process of enclosure in the Western Lowlands. Scottish Geogr. Magazine 62 (3): 100-110, 1946. 28) LEU, FRITZ: Anthropogeographie der Freiberge. Diss. Univ. Basel 1955. 29) MarquardT, G.: Die Schleswig-Holsteinische Knicklandschaft. Schriften des Geogr. Inst. der Univ. Kiel 13 (3), 1950. 30) Merian, MatTh.: Topographia Helvetiae, Rhaetiae et Valesiae: Das ist/Beschreibung unnd eygentliche Abbildung der vornehmsten Stätte und Plätze in der Hochlöblichen Eydgnossschafft/Graubündten/Wallis/und etlicher zugewandten Orthen. Frankfurt a/M 1654. 31) Meyer, KarL-Alfons: La haie, un type forestier du moyen âge. Journal Forestier Suisse (4): 1-6, 1942. 32) NERTZ, RENÉ L.: Die ländlichen Siedlungen der Ajoie. Diss. Univ. Basel 1950. 33) Pfyffer, Kasimir: Der Kanton Luzern. Gemälde der Schweiz. Bd. 3, St. Gallen/Bern 1858. 34) Pulver, Eugen E.: Von der Dreizelgenordnung zur bernischen Kleegraswirtschaft. Diss. ETH Zürich 1956. 35) Schlatter, Th.: Die Pflanzenwelt St. Gallens. Die Stadt St. Gallen und ihre Umgebung, herausg. von Gottlieb Felder: 71-147, St. Gallen 1916. 36) Schmithósen, Josef: Bemerkungen zu dem Problem der Bodenabtragung in der Kulturlandschaft. Angewandte Pflanzensoziologie Heft 15: 165-168, Stolzenau/Weser 1958. 37) SPINNER, HENRI: Le Haut-Jura neuchâtelois nord-occidental. Beitr. zur Geobotan. Landesaufn. Heft 17, Bern 1932. 38) 
Steiner-Haremaker, INGrid: Verbreitung und Bedeutung der Grünhecken in der Schweiz. Diplomarbeit (Manuskr.) Geogr. Inst. Univ. Zürich 1959. 39) Steiner, PeTer: Das Gericht Reinach zur Zeit der Berner Herrschaft. Diss. Univ. Zürich 1956. 40) Timmermane, L.: Das Eupenerland und seine Grünlandwirtschaft. Bonner Geogr. Abh. Heft 5, 1951. 41) Troll, Carl: Heckenlandschaften im maritimen Grünlandgürtel und im Gäuland Mitteleuropas. Erdkunde 5 (2): 152-157, Bonn 1951. 42) Tũxen, Reinhold: Hecken und Gebüsche. Mitt. der Geogr. Ges. Hamburg 50: 85-117, 1952. 43) Uhlig, Harald: Die Kulturlandschaft. Methoden der Forschung und das Beispiel Nordostengland. Kölner Geogr. Arbeiten Heft 9/10, 1956. 44) Vōgeli, Hermanx: Die agrargeographische Gliederung im Übergangsgebiet vom Mittelland zu den Alpen zwischen Linthebene und Alpnachersee. Diss. Univ. Zürich 1958. 45) VolKarT, A.: Dreifelder- und Egartenwirtschaft in der Schweiz. Frauenfeld 1902. 46) Vussseler, Paul: Der Aargauer Jura. Aarau 1928. 47) Vul.liemin, L.: Der Kanton Waat. Gemälde der Schweiz Bd. 19, St. Gallen/Bern 1847. 48) WiLCzeK, E.: La flore des haies en Valais et principalement à Zermatt. Veröff. des Geobotan. Inst. Rübel Heft 3: 264-270, 1925. 49) WindLeR, HaNs: Zur Methodik der geographischen Grenzziehung am Beispiel des Grenzbereiches der Kt. Schwyz, Zug und Zürich. Geogr. Helv. 9 (3): 129-185, 1954. 50) WinkLer, ERNsT: Die schweizerische Kulturlandschaft zur Zeit Johann Stumpfs. Geogr. Helv. 2 (4): 223-228, 1947. 51) Winteler, Rudolf: Studien über Soziologie und Verbreitung der Wälder, Sträucher und Zwergsträucher des Sernftales. Vierteljahresschrift der Naturf. Ges. Zürich 72 (1/2): 1-185, 1927. 52) Wyder, SAmuel: Die Schaffhauser Karten von Hauptmann Heinrich Peyer (1621-1690). Diss. Univ. Zürich 1949.

\section{Karten}

53) Landeskarte der Schweiz $1: 25000$ und 1:50 000. Publizierte Blätter bis Okt. 1958. 54) Karte des Kt. Zürich von Hans KonRad Gyger, 1667. 55) Kulturlandkarte der Schweiz 1:200 000. Herausg. von der Abt. für Landwirtschaft im EVD, Bern 1952-54. 56) Wirtschaftsgeographische Karte der Schweiz 1:300 000, bearbeitet von Hans Carol, Kümmerly \& Frey, Bern 1946. 57) Karte der ländlichen Siedlungen von F. Nussbaum und P. Vosseler, 1931, in J. FrüH: Geographie der Schweiz.

\section{Nicht gedruckte Quellen}

58) Zehntenpläne im Staatsarchiv des Kt. Zürich: No. 1: Adliswil von JoH. MÓller 1787; No. 32: Dübendorf von H. J. HulfTEGger 1681; No. 273 b: Uitikon, Ringlikon und Urdorf von Joh. RUD. WerdmOller 1680 ; No. 305: Guntalingen von P. Josepho 1739, ergänzt von CASPAR ObRST 1813. 59) Haupturbar der Grafschaft Lenzburg 1667-1677 im Staatsarchiv des Kt. Aargau.

\section{L'EXTENSION ET LA PORTÉE GÊGRAPHIQUE DES HAIES VIVES EN SUISSE}

Le présent article analyse l'extension et la portée géographique des haies en Suisse, dont on ne fait que rarement mention. En se basant sur la Carte nationale $1: 25000$ et $1: 50000$, nous avons élaboré une carte de densité (voir fig. 1) faisant ressortir les contrées où les haies sont le plus fortement répandues: le Jura, l'ouest du Plateau, les zones subalpines et préalpines ainsi que quelques vallées des Alpes centrales. Le type de haies est étroitement lié à la structure économique. Fig. 2 montre l'étendue des haies dans les différentes zones économiques et d'habitat. En principe, nous pouvons distinguer entre deux genres de haies: les haies sauvages et les haies plantées. Les haies sauvages sur des rideaux ou de pierriers se trouvent surtout dans le Jura tabulaire (Randen y compris), en Ajoie, dans les Franches-Montagnes, dans les chaînes occidentales du Jura, dans l'ouest du Plateau et dans les vallées des Alpes et des Préalpes. Par contre, nous rencontrons le plus souvent les haies plantées en bordure de propriétés privées ou communes ou entre des parcelles à exploitation différente en Ajoie, dans l'est des chaînes du Jura, dans le canton de Genève ainsi que dans les régions subalpines et préalpines. Les haies plantées, existant encore de nos jours, sont les vestiges de l'ancien paysage bocager et permettent de tirer des conclusions historiques quant à la situation foncière et économique. Dans les contrées où l'économie rurale collective (assolement triennal ou biennal) était dominant, les haies servaient à délimiter les parcelles exploitées individuellement (voir fig. 4, «semibocage»). Dans les anciennes régions à assolement avec pâtures temporaires (habitat dispersé prépondérant), les haies bordaient les propriétés arrondies qui étaient en outre souvent subdivisées en différentes parcelles («plein bocage»). Si nous trouvons encore aujourd'hui des haies naturelles au milieu de prés, nous pouvons admettre qu'autrefois il y existait une plus grande étendue de champs ou de vignobles. 